\title{
Electronic spectra of polyatomic molecules with resolved individual rotational transitions: Benzene
}

\author{
E. Riedle, H. J. Neusser, and E. W. Schlag \\ Institut für Physikalische Chemie der Technischen Universität München, Lichtenbergstrasse 4, D-8046 \\ Garching, West Germany \\ (Received 18 May 1981; accepted 29 June 1981)
}

\begin{abstract}
The density of rotational transitions for a polyatomic molecule is so large that in general many such transitions are hidden under the Doppler profile, this being a fundamental limit of conventional high resolution electronic spectroscopy. We present here the first Doppler-free cw two-photon spectrum of a polyatomic molecule. In the case of benzene, 400 lines are observed of which 300 are due to single rotational transitions, their spacing being well below the Doppler profile. The resolution so achieved is $1.5 \times 10^{\text {? }}$. Benzene is a prototype planar molecule taken to have $D_{6 h}$ symmetry in the ground as well as in the first excited state. From our ultra-high resolution results it is found that benzene in the excited $S_{1}$ state is a symmetrical rotor to a high degree. A negative inertial defect is found for the excited state. The origin of this inertial defect is discussed.
\end{abstract}

\section{INTRODUCTION}

Rotational spectroscopy has been a prime source for the accurate determination of molecular structures. In an electronic spectrum the observation of rotational structure requires the highest resolution. For a large molecule the density of states is generally so high that many rotational states are grouped even in high resolution, this being limited by the Doppler profile of the transition. This is particularly so for polyatomic systems. Hence, all, even high resolution, spectra of polyatomic molecules have only produced rotational envelopes, ${ }^{1}$ i.e., groupings of rotational transitions. This has greatly limited all structural information so far obtainable from molecular electronic spectroscopy.

One recent method designed to observe individual lines is not based on an increase in resolution, but rather on a substantial reduction in the number of possible rotational transitions by freezing the molecule in a supersonic jet. ${ }^{2}$ The total number of rotational transitions is drastically reduced by this technique, but it does not follow that the average spacing of the remaining lines is necessarily increased. This is in general only so if neighboring lines have strongly varying Boltzmann factors in the course of the spectrum, e.g., if those lines originate from strongly interleaving rotational branches. More severely, only transitions of low $J$ are measured. These are in general not useful for a precise determination of moments of inertia and therefore contain only moderate structural information. We will show for our example that for an accurate structural determination the observation of lines with $J$ at least equal to $J=9$ is necessary.

Many other benefits are derived from an increase in resolution above conventional high resolution spectra, e.g., also, very small splittings of degenerate lines which are expected due to a slight change in symmetry in the excited electronic state can be observed, an essential application in this work.

In this work for the first time as many as 400 individual rotational transitions are reported for a polyatomic molecule (benzene). We performed a Doppler-free twophoton measurement of the polyatomic molecule benzene, with two cw lasers, employing a slightly different wavelength in each laser. It is shown for the first time that well defined single rotational transitions can be observed in the room temperature electronic spectrum of a polyatomic molecule as large as benzene. Levels up to $J \sim 30$ were seen in a spectrum continuing over more than $100 \mathrm{GHz}$ with a resolution of some $80 \mathrm{MHz}$. It will be shown that the analysis of this spectrum yields detailed information about the structure of this prototype molecule in the excited state which has so far not been available from highest resolution, but Doppler-limited, UV spectroscopy.

\section{EXPERIMENTAL}

A scheme of the experimental setup is presented in Fig. 1. Two-photon spectra of benzene molecules with a sub-Doppler resolution are here obtained in a two laser experiment. One laser is a Coherent $599 \mathrm{cw}$ single mode dye laser with an output power of some $100 \mathrm{~mW}$

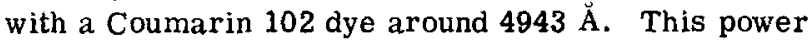
level is too low for an efficient two-photon absorption in benzene, since in a molecule of this size the oscillator strength of the electronic transition is not concentrated within a single line as in the case of atoms, but rather is distributed over many thousands of rovibronic lines. Therefore, a Coherent $\mathrm{cw} \mathrm{Ar}{ }^{*}$ ion laser as a second laser of high power is used, providing the second photon of fixed energy for the two-photon process. This $\mathrm{Ar}^{+}$ion laser is operated with a temperature-controlled internal etalon at the $5145 \AA$ line in the single mode. The output power is about $300 \mathrm{~mW}$, which increases the two-photon absorption probability by a factor of 30 and makes it possible to scan the two-photon spectrum with a reasonable signal to noise ratio.

The two counterpropagating laser beams are carefully alligned and brought to a common focus within a vacuum cell containing benzene vapor. The vapor pressure was adjustable to particular values in the range of 4.3 to 60 Torr using various low temperature liquid baths.

After the benzene molecules have been excited by the two-photon absorption, they undergo fluorescence relaxation with a quantum yield of about $20 \%$, at the excita- 


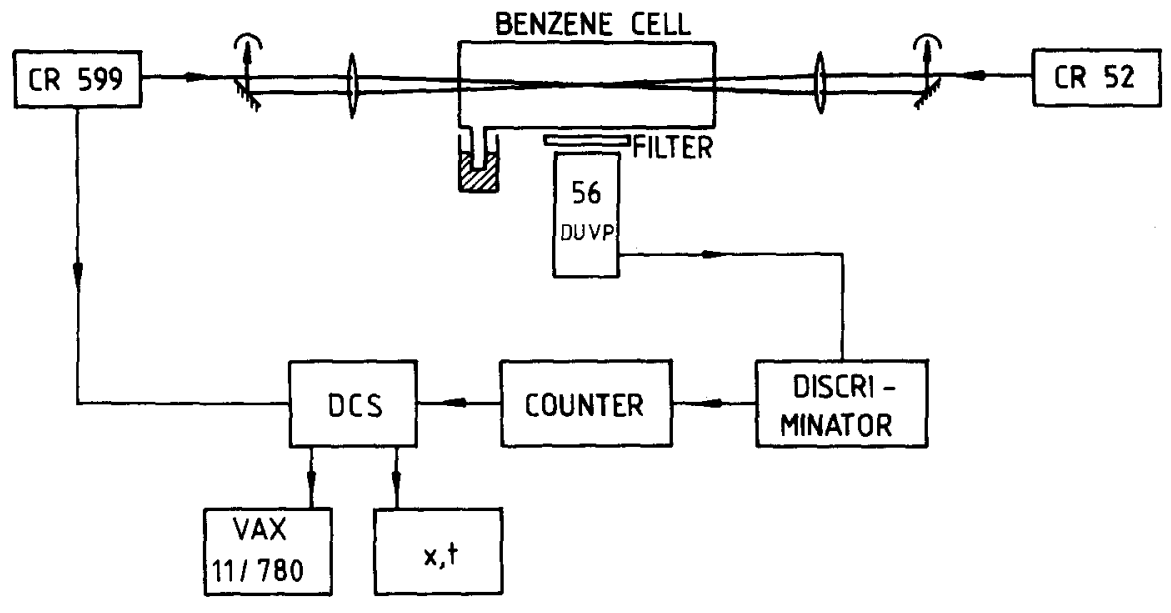

FIG. 1. Scheme of the experimental setup for measuring sub-Doppler twophoton spectra of benzene molecules with two $\mathrm{cw}$ lasers.

tion energy of this experiment. ${ }^{3}$ The corresponding UV fluorescence is monitored with a photomultiplier and photon counted. The counting rate is recorded on a strip chart recorder as a function of the photon energy of the scanning single mode dye laser. At the same time the raw counting rate is directly transfered to a DCS 102 data processing system, stored on floppy discs, and then transfered to a VAX 11/780 computer. All data are processed in the computer system and finally plots of the complete two-photon spectrum with suitable scaling factors are obtained made up from several overlapping scans of the dye laser.

During the course of the experiment the laser scan as well as the frequency of the fixed wavelength laser are assured by two external etalons. Only those scans have been used for the analysis of the spectrum that show neither a mode jump of the dye nor of the $\mathrm{Ar}^{+}$ion laser. The absolute accuracy of the frequency scale in the dye laser scan is then given by the linearity of the dye laser scan and by the accuracy of the external etalon and is therefore better than $0.5 \%$ of the scan range.

The spectral resolution of our system is limited to $45 \mathrm{MHz}$ due to several factors. There is a residual Doppler width firstly due to the slight energy mismatch of $796 \mathrm{~cm}^{-1}$ of both laser photons and secondly due to a small angle between both laser beams.

Furthermore, the linewidth of the $\mathrm{Ar}^{+}$ion laser is 10 $\mathrm{MHz}$ since this laser is not stabilized. As will be shown below, the smallest linewidth measured in the spectrum is some $70 \mathrm{MHz}$ and therefore larger than produced by the experimental resolution of our setup. This difference is due to the collisional broadening at the lowest pressures possible in this experiment ( 4 Torr).

Although resolution is somewhat limited by the residual Doppler width in the two laser experiment, there is an important advantage of this type of experiment. When using two photons of equal frequency there is, besides the Doppler-free spectrum, always a Dopplerbroadened background which is due to the simultaneous absorption of two photons from one laser beam. For an atomic $s-s$ transition with the selection rule $\Delta m=0$, this Doppler-broadened component certainly disappears using (in the atomic frame) counter-circularly polarized photons ${ }^{4}$ since the absorption of two photons circularly polarized in the same sense is not possible in these experiments. The situation, however, is more complicated in molecular spectroscopy. In such cases, except for the rare case of spherical top molecules, the anisotropic component of the two-photon absorption tensor always leads to an absorption for two photons circularly polarized in the same sense. This may be six times the absorption produced by two counter-circularly polarized photons from counterpropagating laser beams for nontotally symmetric two-photon transitions. ${ }^{5}$ Hence, a Doppler-broadened background cannot be avoided in most molecular systems by the method so useful in atomic spectra of just using counter-circularly polarized light beams. In polyatomic molecules this background becomes a severe problem due to the many rotational transitions located within the Doppler width. In a two color experiment one is able to eliminate the Doppler-broadened background if the resonance condition is not fulfilled for absorption of two photons from one laser beam. This has been carefully checked in our experiment. Absorption of two photons from the tunable dye laser beam generally is not critical because of the low power of $10 \mathrm{~mW}$. A more severe problem is the absorption of two photons from the $\mathrm{Ar}^{*}$ ion laser. When using the $5145 \AA$ A line, the energy of two photons is not sufficient for an electronic excitation starting from the zero ground state level and therefore two-photon absorption is in the very weak hot band region of the two-photon spectrum. ${ }^{6}$ Hence, there is only a constant $10 \%$ background in the observed spectrum due to this effect.

\section{RESULTS AND DISCUSSION}

In Fig. 2 a Doppler-free scan of benzene over $4 \mathrm{~cm}^{-1}$ with a resolution of $80 \mathrm{MHz}$ is demonstrated, indicating some 400 lines. This spectrum is composed of six individual overlapping experimental scans. Even though each individual scan is limited to $30 \mathrm{GHz}$ due to the technical characteristics of the Coherent $599 \mathrm{cw}$ dye laser, a continuous precise frequency scale over 130 $\mathrm{GHz}$ is obtained. This spectrum reflects the extremely resolved blue edge of the $Q$ branch $(\Delta J=0)$ of the strongest vibrationally induced $\left(14_{0}^{1}\right) S_{1}-S_{0}$ transition in $\mathrm{C}_{6} \mathrm{H}_{6} \cdot{ }^{6}$ 

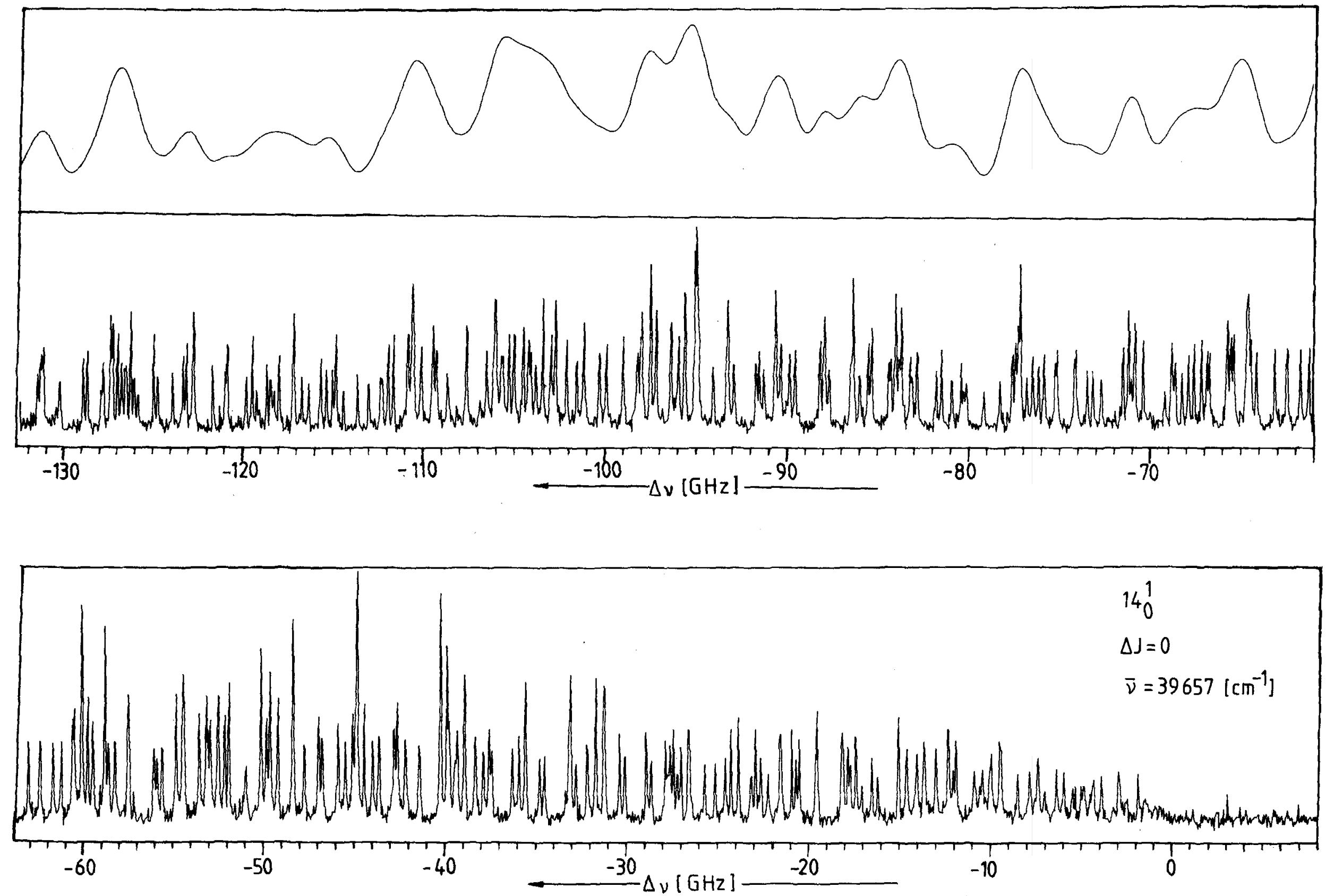

FIG. 2. The total measured range of the Doppler-free two-photon spectrum of $\mathrm{C}_{6} \mathrm{H}_{6}$ (lower traces). The upper trace represents the highest resolution possible in conventional Doppler-limited spectroscopy. It was obtained by folding the spectrum in the middle trace with the Doppler width of $1.7 \mathrm{GHz}$. 
For comparison and for demonstration of the ultra-high resolution in the upper part of Fig. 2, the corresponding part of the spectrum between -60 and $-130 \mathrm{GHz}$ is shown when folded with the Doppler width of $1.7 \mathrm{GHz}$, the highest resolution hitherto obtainable. This transition is induced by the vibration $v_{14}$ of $b_{2 u}$ symmetry ${ }^{7}$ in the $S_{1}$ electronic state of $B_{2 u}$ symmetry. Therefore, the transition tensor moment transforms as the totally symmetric representation of the $D_{8 h}$ point group, and for linearly polarized light the isotropic tensor contribution dominates the anisotropic tensor contribution ${ }^{6}$ in the $Q$ branch as well as in the remaining four branches with $\Delta J= \pm 1, \pm 2$. Because of the $A_{1 g}$ transition symmetry, there is a $\Delta K=0$ selection rule for the projection $K$ of $J$ on the figure axis of the symmetric top.

Because of the dominant isotropic contribution to the intensity, we may neglect all contributions from other branches within the frequency interval under investigation. This simplifies the analysis of the spectrum.

\section{A. Qualitative analysis}

At first sight, from the result in Fig. 2 it is obvious that the vibronic spectrum of benzene, which is normally a band type spectrum, is now resolved and represents sharp line character such as typically observed for much simpler molecules with smaller moments of inertia. Indeed, as the detailed rotational analysis will show, most of the lines in the spectrum represent single rotational lines. The line character of this ultra-high resolution spectrum makes the assignment and analysis of the spectrum a straightforward procedure.

Benzene in its electronic ground state is known to be a planar symmetric rotor with a sixfold rotational axis being the figure axis of the oblate symmetric top. There have been extensive studies concerning the geometry of this prototype molecule in the first electronically excited state. ${ }^{8}$ Within the model of simple molecular orbital theory, this first electronically excited state is due to the promotion of one bonding electron to an antibonding orbital. There has been a long discussion as to whether the $\pi \pi^{*}$ promotion and the decreasing of bond energy lead to a change in structure in the excited state. So far, high resolution but Doppler-limited UV measurements have shown that there is an increase in $\mathrm{C}-\mathrm{C}$ bond length in the first excited state. In all previous work it has been assumed that benzene is a planar molecule, for the ground as well as the first excited electronic states. Previous vibronic and rotational analysis of high resolution spectra ${ }^{8,9}$ have been consistent with this assumption, but it must be remembered that due to the high rotational line density, even these high resolution measurements only reflect rotational envelopes over many individual transitions. This is illustrated by the upper part of Fig. 2. Here the measured spectrum between -60 and $-130 \mathrm{GHz}$ (middle trace of Fig. 2) has been folded with the Doppler width of $1.7 \mathrm{GHz}$. Hence, for the assignment of the spectrum we assumed initially a planar oblate symmetric top structure in the excited state and start from the corresponding energy equation. The energy displacement $\Delta E$ from the pure vibronic transition frequency is then given by ${ }^{10}$

$$
\Delta E=\left(B^{\prime \prime}-B^{\prime}\right)\left[J(J+1)-\frac{1}{2} K^{2}\right],
$$

where $A^{\prime \prime}=B^{\prime \prime}=2 C^{\prime \prime}\left(A^{\prime}=B^{\prime}=2 C^{\prime}\right)$ are the rotational constants of the symmetric top in the electronic ground (excited) state. $I_{A}=I_{B}=1 / B=1 / A$ and $I_{c}=1 / C=2 I_{A}$ are the moments of inertia along the principal axes of the molecule. It is easily seen that the planarity condition for a symmetric top

$$
I_{c}-I_{A}-I_{B}=0
$$

is fulfilled for the above assumptions. On the basis of Eq. (1), we made a computer calculation of the corresponding part of the spectrum. The relative line intensities have been obtained taking into account the population of the rotational ground state, the $M$ degeneracy, and the statistical weights of the transitions due to nuclear spin statistics. ${ }^{11}$ Here the same rotational constants $B^{\prime \prime}$ and $B^{\prime}$ are taken $\left(B_{0}^{\prime \prime}=0.18960 \mathrm{~cm}^{-1},{ }^{12} B_{v}^{\prime}\right.$ $=0.18134 \mathrm{~cm}^{-19}$ ), as for simulation of recent Dopplerlimited two-photon measurements ${ }^{\theta}$ of the same vibronic band which are the most accurate measurements known from the literature. The resulting lines are then folded with a Gaussian profile of $90 \mathrm{MHz}$ half-width yielding the best agreement with the measured spectrum. A plot of this calculated spectrum is shown in Fig. 3(a). This spectrum in Fig. 3(a) then reflects the computed spectrum of Ref. 9 that was thought to give the best fit to the Doppler-limited experimental results. It is plotted on a 20 times expanded scale. Figure $3(\mathrm{~b})$ shows our ultra-high resolution results for comparison. A comparison of both spectra shows rough qualitative agreement in the gross features, such as line positions and strengths at the blue side of the spectrum made up by transitions with low $J$ and $K$. The identifications of all lines up to $30 \mathrm{GHz}$ starting from the 00 rotationless level is readily possible. It is quite apparent that the scaling of the computed spectrum does not accurately fit the measured spectrum, indicating already at this point a discrepancy in the rotational constants of Ref. 9 . There is, however, one further and fundamental disagreement between this calculation and the Dopplerfree measurements.

From Eq. (1) it can be seen that for a planar symmetric rotor there are particular transitions with $J_{i}$, $K_{j}$ accidentally degenerate in energy with other transitions $J_{k}, K_{l}$ independent of the value $B^{\prime \prime}-B^{\prime}$. For example, the transitions with $J=9, K=4$ and $J=11, K=10$, with the intensity ratio of $0.82: 1$, according to Eq. (1) have the same energy. The corresponding region of the measured spectrum is around $20 \mathrm{GHz}$ on the red side of the band origin. These transitions as well as three other examples are marked in Fig. 3(a). A closer inspection unambiguously shows that in the measured spectrum of Fig. 3(b) the degenerate line $J=9, K=4$, and $J=11, K=10$ is split into its two components separated by $0.19 \mathrm{GHz}$. This is seen from the measured intensity ratio of $0.75: 1$. A similar splitting is also observed for the other examples shown in Fig. 3. From this splitting it is clear that the simple energy relation of the symmetric rotor [Eq. (1)] is in disagreement with 


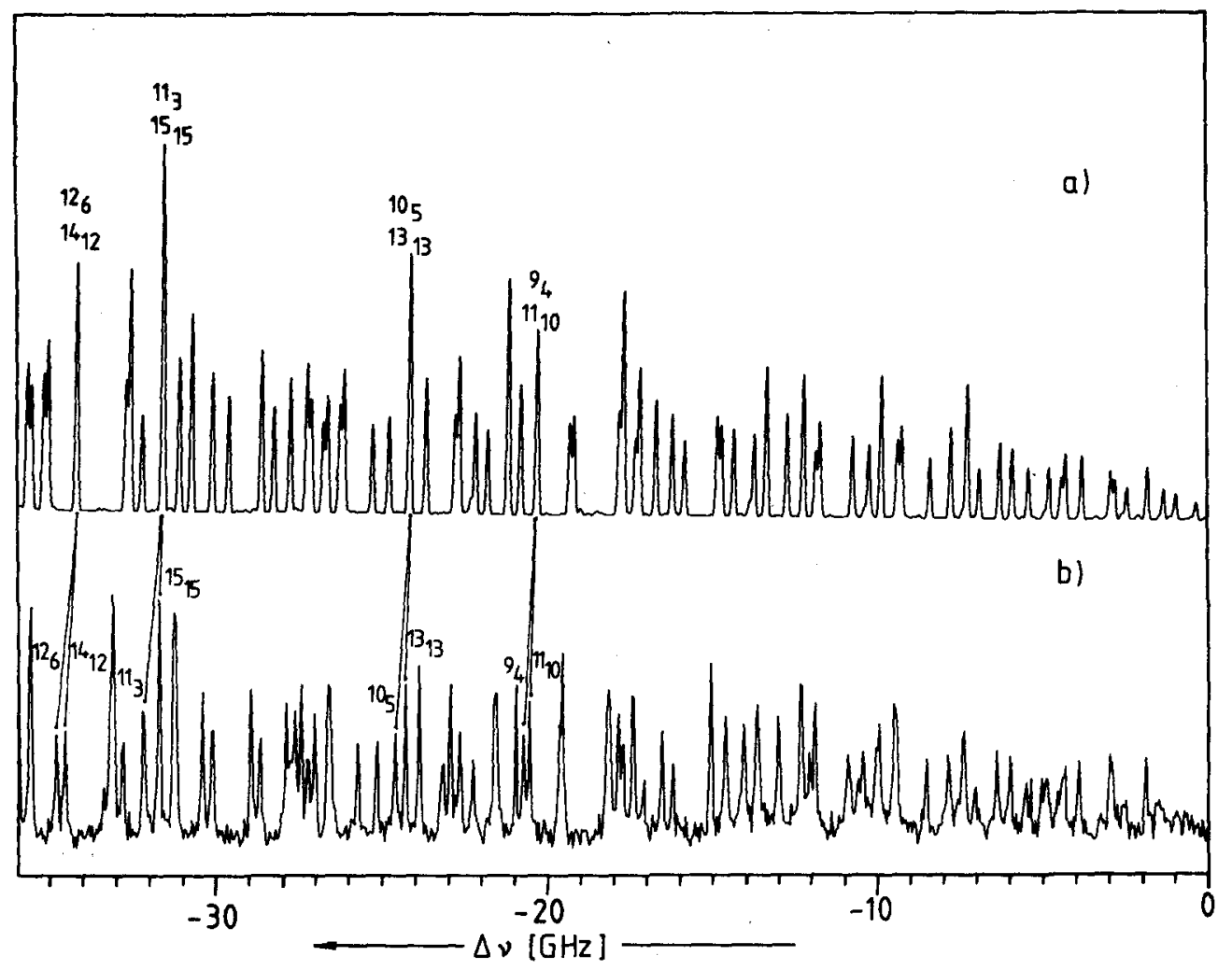

FIG. 3. The extremely resolved band edge of the $Q$ branch in the $14_{0}^{1}$ two-photon transition of $C_{6} \mathrm{H}_{6}$. (a) As calculated from the rotational constants found from the hitherto best resolved but Doppler-limited experiment of the same band in Ref. 9 . (b) As measured in our Doppler-free experiment with a resolution of $80 \mathrm{MHz}$. Several transitions with different $J_{K}$ which are degenerate in (a) under the assumption of planarity $\left(C=\frac{1}{2} B\right)$ are found to be split into their two components in the measured spectrum.

our experimental results. Since the numerical values of $B^{\prime \prime}$ and $B^{\prime}$ do not in any way affect the degeneracy of both transitions under investigation, we may conclude that the $J, K$ dependence of the energy in Eq. (1) is not correct. Two possible effects come to mind which modify the $J$ and/or $K$ dependence and could explain the line splitting.

(i) The first possibility would be the centrifugal distortion of the molecule due to the rapid rotation of the molecule at high $J, K$ levels. When centrifugal distortion is active, new terms in the energy formula appear depending on $J^{2}(J+1)^{2}, J(J+1) K^{2}$, and $K^{4}$ with the centrifugal distortion constants $D_{J}, D_{J K}$, and $D_{K}$. According to Ref. 13, an upper limit of the centrifugal distortion constant $D_{J}$ can be found from the lowest frequency vibrations of $a_{1 g}$ and $e_{2 g}$ symmetry. This yields an upper limit of $D_{f}^{\prime \prime}=5.1 \times 10^{-8} \mathrm{~cm}^{-1}$ for the ground state and $D_{J}^{\prime}=6.5 \times 10^{-8} \mathrm{~cm}^{-1}$ for the electronically excited state. Taking into account that $D_{J}, D_{K}$, and $D_{J K}$ are related for a planar symmetric top ${ }^{14,15}$ and that in the electronic spectrum only differences of ground state and excited state centrifugal distortion constants appear, we calculate an upper limit for the splitting which could be due to centrifugal distortion of only 7.7 MHz of transitions $J=9, K=4$ and $J=11, K=10$. This maximal effect is more than one order of magnitude smaller than our observed result of $190 \mathrm{MHz}$.

(ii) A second explanation then would be that the as sumption $C^{\prime}=\frac{1}{2} B^{\prime}$, yielding the simple $J, K$ dependence in Eq. (1), is not correct, i.e., that there is an inertial defect $\left(I_{C}-I_{B}-I_{A} \neq 0\right)$ in either the ground or the electronically excited state or in both. The simple energy dependence of Eq. (1) then must be changed to the general case of a rigid nonplanar symmetric top ${ }^{10}$

$$
\Delta E=\left(B^{\prime \prime}-B^{\prime}\right)[J(J+1)]+\left(C^{\prime \prime}-C^{\prime}-B^{\prime \prime}+B^{\prime}\right) K^{2} .
$$

The spectrum is now characterized by two independent rotational constants $B$ and $C$.

\section{B. The rotational constant $B^{\prime}$}

In order to display this result in a quantitative manner we cannot employ the results for $B^{\prime}$ obtained from literature data involving Doppler-limited experiments since these have been obtained under the assumption that benzene is a planar symmetric top in the electronically excited state, i. e. , $C^{\prime}=\frac{1}{2} B^{\prime}$. In our experiment we are resolving single rotational lines and hence an independent analysis for $B^{\prime}$ and $C^{\prime}$ becomes a much simpler and reliable procedure. To ascertain $B^{\prime}$ we measure the accurate frequency position of lines with high $J$ and low $K(K=0,1)$. These line positions are independent of $C^{\prime}$. Lines with $K=1$ and to some degree also $K=0$ can be well identified in our Doppler-free spectrum up to $J=21$. This would be a hopeless problem in Doppler-limited spectroscopy. From several independent line position measurements we then obtain

$$
B_{0}^{\prime \prime}-B_{v}^{\prime} \approx 0.00842 \pm 0.00002 \mathrm{~cm}^{-1} \text {. }
$$


The error limits represent the largest deviations found in several independent measurements. This then represents a new value for $B_{v}^{\prime}$, being outside the error limits of the most accurate value of $B_{v}^{\prime}=0.00826 \pm 0.00001$ hitherto found from Doppler-limited measurements of the same vibronic state. ${ }^{9}$ This former value, however, has been found assuming planarity for $\mathrm{C}_{6} \mathrm{H}_{8}$ in the excited state and this might be the reason for the relatively large discrepancy.

From our result for $B^{\prime \prime}-B^{\prime}$ and from the most accurate recently reported ground state value of $B_{0}^{\prime \prime}$ $=0.189751 \pm 0.000025 \mathrm{~cm}^{-118}$ we then obtain for the excited state a value of

$$
B_{y}^{\prime}=0.18133 \pm 0.000045 \mathrm{~cm}^{-1} \text {. }
$$

It is interesting to note here that the accuracy of this excited state value reflects the accuracy of the ground state value in the literature. The accuracy of the measurement reported here is higher, but only refers to the difference between the two inertial constants. The index $v$ indicates that the measured value corresponds to the vibrationally excited state $14^{1}$ rather than to the vibrationless electronically excited state. The variation of the difference $B_{v}-B_{0}$ in the rotational constants due to an excited vibration in the benzene molecule is known to be typically $2 \times 10^{-4} \cdot 17,18$

\section{The rotational constant $C^{\prime}$}

With this accurate value for $B_{v}^{\prime}$ we are able to deduce an accurate value for $C^{\prime \prime}-C^{\prime}$ from our experiments. This was done by measuring the splitting of several line pairs that are degenerate in the planar symmetric top approximation. This is the most accurate way to determine $\Delta C$ since the line splitting itself is independent of $\Delta B$ and since the frequency distance of the two lines is measured with high accuracy. The measured line splittings are summarized in Table I together with the individually calculated values of $\Delta(C-B)$.

This then yields an average value of

$$
\Delta(C-B)=-0.00428 \pm 0.00001 \text {, }
$$

whereas for an exactly planar molecule one would find

$$
\Delta(C-B)=-0.00421 \text {. }
$$

Finally, we obtain a value for $C_{v}^{\prime}$ in the electronically and vibrationally excited state under the assumption that benzene is perfectly planar in the ground state $\left(C_{0}^{\prime \prime}\right.$

TABLE I. Several transitions $J_{K}$, which should be degenerate for a planar $\mathrm{C}_{6} \mathrm{H}_{6}$ molecule, and the experimentally observed splittings $\delta E$ of these lines. Also given are the values $\Delta(C-B)$ calculated from the splittings and the experimentally obtained value of $\Delta B$.

\begin{tabular}{cccc}
\hline $\begin{array}{c}\text { Degenerate } \\
\text { lines } J_{K}\end{array}$ & $\begin{array}{c}\text { Observed splitting } \\
\delta E(\mathrm{GHz})\end{array}$ & $\Delta(C-B)\left(\mathrm{cm}^{-1}\right)$ \\
\hline $9_{4}$ & $11_{10}$ & 0.19 & -0.0042854 \\
$10_{5}$ & $13_{13}$ & 0.36 & -0.0042933 \\
$11_{3}$ & $15_{15}$ & 0.48 & -0.0042841 \\
$12_{6}$ & $14_{12}$ & 0.25 & -0.0042872 \\
\hline
\end{tabular}

TABLE II. Rotational constants $B$ and $C$ and structural parameters of $\mathrm{C}_{6} \mathrm{H}_{6} . \quad r_{\mathrm{CH}}$ is the $\mathrm{C}-\mathrm{H}$ bond length, $r_{\mathrm{CC}}$ the $\mathrm{C}-\mathrm{C}$ bond length, and $h / 2$ is the displacement of $C$ atoms from the ground state molecular plane. $\alpha$ is the angle between the bent planes of the molecule. Ground state values are taken from the literature, and excited state values represent effective values from this lwork.

\begin{tabular}{lll}
\hline & \multicolumn{2}{c}{ Electronic state } \\
\cline { 2 - 3 } & Ground $\left(S_{0}\right)$ & \multicolumn{1}{c}{$\begin{array}{l}\text { Observed excited } \\
\left(S_{1} ; 14^{1}\right)\end{array}$} \\
\hline Symmetry & $D_{6 h}$ & $D_{3 d}$ \\
$B\left(\mathrm{~cm}^{-1}\right)$ & $0.189751 \pm 0.000025^{2}$ & $0.18133 \pm 0.000045$ \\
$C\left(\mathrm{~cm}^{-1}\right)$ & $C^{\prime \prime}=\frac{1}{2} B^{\prime \prime}$ & $0.09074 \pm 0.00008$ \\
$r_{C H}(\AA)$ & $1.084^{\mathrm{b}}$ & $1.084^{\mathrm{c}}$ \\
$r_{\mathrm{CC}}(\AA)$ & $1.397^{\mathrm{b}}$ & $1.4323 \pm 0.0003$ \\
$h(\AA)$ & 0 & $0.043 \pm 0.004$ \\
$\alpha(\mathrm{deg})$ & 0 & $5.2 \pm 0.5$ \\
\hline \hline
\end{tabular}

${ }^{a}$ Reference $16 . \quad{ }^{b}$ Reference 12 . 'Reference 9 .

$\left.=\frac{1}{2} B_{0}^{\prime \prime}\right)$. Therefore, $C_{v}^{\prime}=-\Delta(C-B)-\frac{1}{2} B_{0}^{\prime \prime}+B_{v}^{\prime}$ and this yields

$$
C_{\nu}^{\prime}=0.09074 \pm 0.00008 \mathrm{~cm}^{-1}
$$

This yields an inertial defect of -0.154 [amu $\left.\AA^{2}\right]$. All molecular constants derived from the analysis of the Doppler-free spectrum in Fig. 1 are summarized in Table II.

\section{Computer simulation of the spectrum}

With the values for $\Delta C$ and $\Delta B$ we are now able to perform a computer simulation of the measured spectrum. The result up to $-70 \mathrm{GHz}$ is shown in Fig. 4 (upper trace) and can be directly compared with the experimental result in Fig. 4 (lower trace). There is a very good agreement of the line positions and line intensities for some 400 transitions covering a range of $4 \mathrm{~cm}^{-1}$ starting from the rotationless transition and including levels with $J$ as high as 31 . This agreement is demonstrated in Fig. 4 for $K$ structures from two different $J$ levels $(J=16$, $J=10$ ). The difference between any particular calculated and measured line position is at most half a linewidth. This strongly confirms the values for $\Delta C$ and $\Delta B$ which have been found not from the simulation of the complete rotational envelope but from the frequency positions of several particular lines (see Table I).

Real discrepancies of measured and calculated spectra, e.g., additional single lines, and disappearance and interchange of neighboring lines, are found only at a few positions for transitions with high $J$ (above 20) and high $K$. These discrepancies are very irregular and they are observed for only about 20 transitions out of 400 assigned and analyzed transitions. Therefore, they cannot be explained by a centrifugal distortion which yields generally a smaller and smoother shift of the 


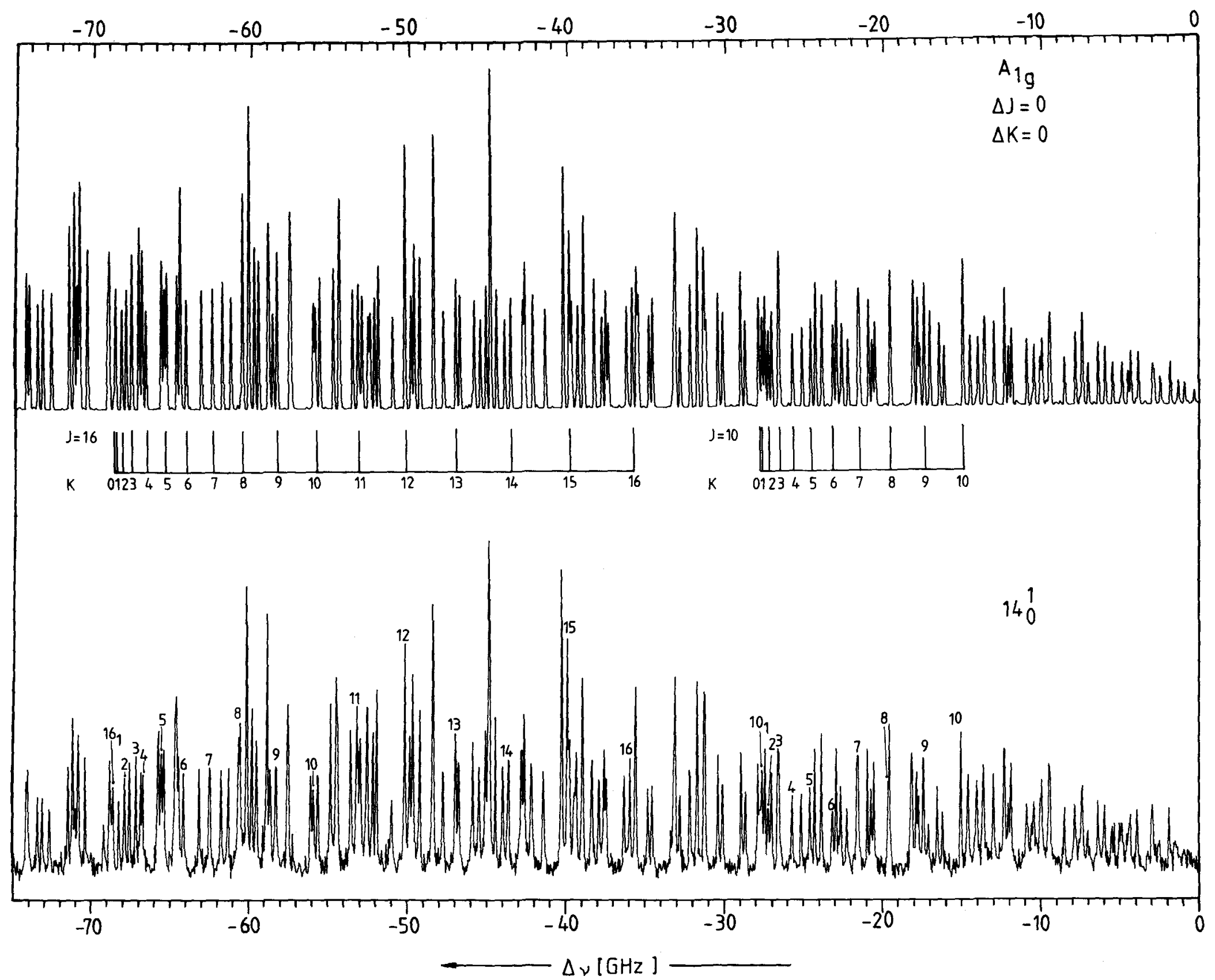

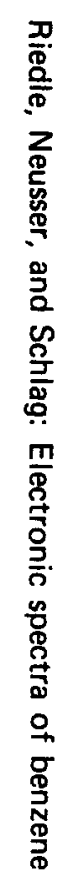

FIG. 4. Measured (lower trace) and calculated (upper trace) spectra of the blue edge of the $Q$ branch of the $14_{0}^{1}$ band. The calculation was performed with the rotational constants in Table II found in this work from the positions of selected lines. The $K$ structures from $J=16$ and $J=10$ levels are marked in both spectra. 
lines. This has been explicitly tested by a variation of the centrifugal distortion constants $\Delta D_{J}, \Delta D_{j k}$, and $\Delta D_{K}$ in the computer plot within the reasonable limits estimated above. From these results we conclude that here we observe rotational perturbations hitherto only observed precisely for much smaller molecules. These perturbations are known to originate from accidental energy matching of the interacting states. Two possible reasons have to be considered for these perturbations. The first is a Fermi resonance between two vibrational states of the same symmetry in the $S_{1}$ state and secondly a Coriolis interaction between two vibrational states of particular symmetry. At first sight, one would suppose a Coriolis coupling between vibrations to be responsible for the perturbation since this effect is found only for the highest $J, K$ levels in the measured spectrum. Further work is required to elucidate this mechanism.

\section{E. Test of asymmetry}

So far, all results interpreted here are based on a symmetric top model of the benzene molecule although it is nonplanar in the excited state. There is a reasonable agreement of measured and calculated spectrum up to $J \approx 20$ and therefore there has been no reason to assume an asymmetry in the assignment as given so far. The procedure of analysis, discussed above, is, however, insensitive to an asymmetry of the molecule. To clarify this open question we especially tested the symmetric top behavior of the molecule.

Asymmetric top theory shows that the $K$ degeneracy due to the $K^{2}$ term in the energy of the symmetric top is lifted when $A \neq B$ and two levels appear with the two pseudo-quantum numbers $K_{-1}$ and $K_{+1}$ related to the two limiting cases of prolate and oblate symmetric tops, respectively.

For fixed asymmetry the splitting $\delta E$ is largest for $K=1$ and in the first order approximation increases quadratically in $J$ according to ${ }^{10}$

$$
\delta E \approx \frac{1}{2}(A-B) J(J+1) .
$$

On the othe $r$ hand, for a given $J$ the splitting decreases strongly with increasing $K$. For a slight asymmetry the splitting is very difficult to observe in the conventional high resolution Doppler-limited spectrum, which integrates over several lines. The Doppler-free scan with single rotational lines provides here a decisive advantage. By comparison of the calculated spectrum against the measured spectrum (Fig. 4) we are able to assign the rotational transitions with $K=1$ up to $J=21$. No splitting of these lines with $K=1$ is observed within the experimental resolution. This is shown for several $J$ values in Fig. 5. From Eq. (3), then, it immediately follows that

$$
\Delta(A-B) \lesssim 1.5 \times 10^{-5} \mathrm{~cm}^{-1} .
$$

This means that $\Delta A=\Delta B$ with very high accuracy. This accuracy is even larger than that for the absolute value of $B$. If we now assume that benzene is a perfectly symmetric top in the ground state, then this demands that

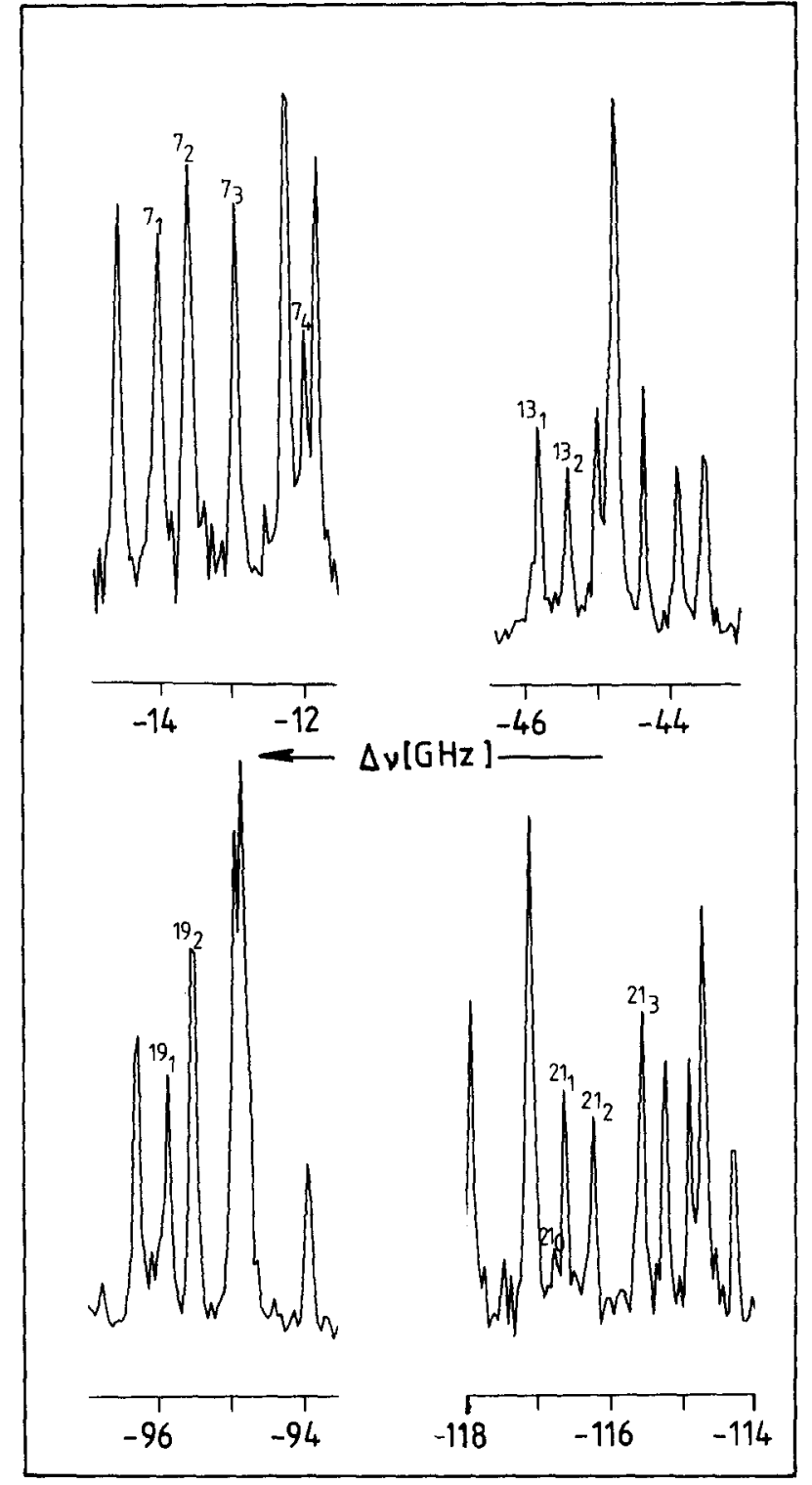

FIG. 5. Four different parts of the measured spectrum showing $J_{K}$ transitions with low $K(K=1,2,3)$ and high $J(J=7,13$, $19,21)$. The origin for all frequency scales is the rotationless transition.

benzene also be a perfect symmetric top in the excited state $14^{1}$.

There is no experimental evidence for any asymmetry from infrared and Raman measurements in the ground state although these measurements are not too sensitive to an asymmetry of the molecule. More precise measurements of the ground state would here be highly desirable.

\section{CONCLUSION}

This is the first case of a polyatomic molecule in which individual rotational transitions have been uncovered far underneath the Doppler profile in the electronic spectrum. This was done for an ambient gas at room temperature. 

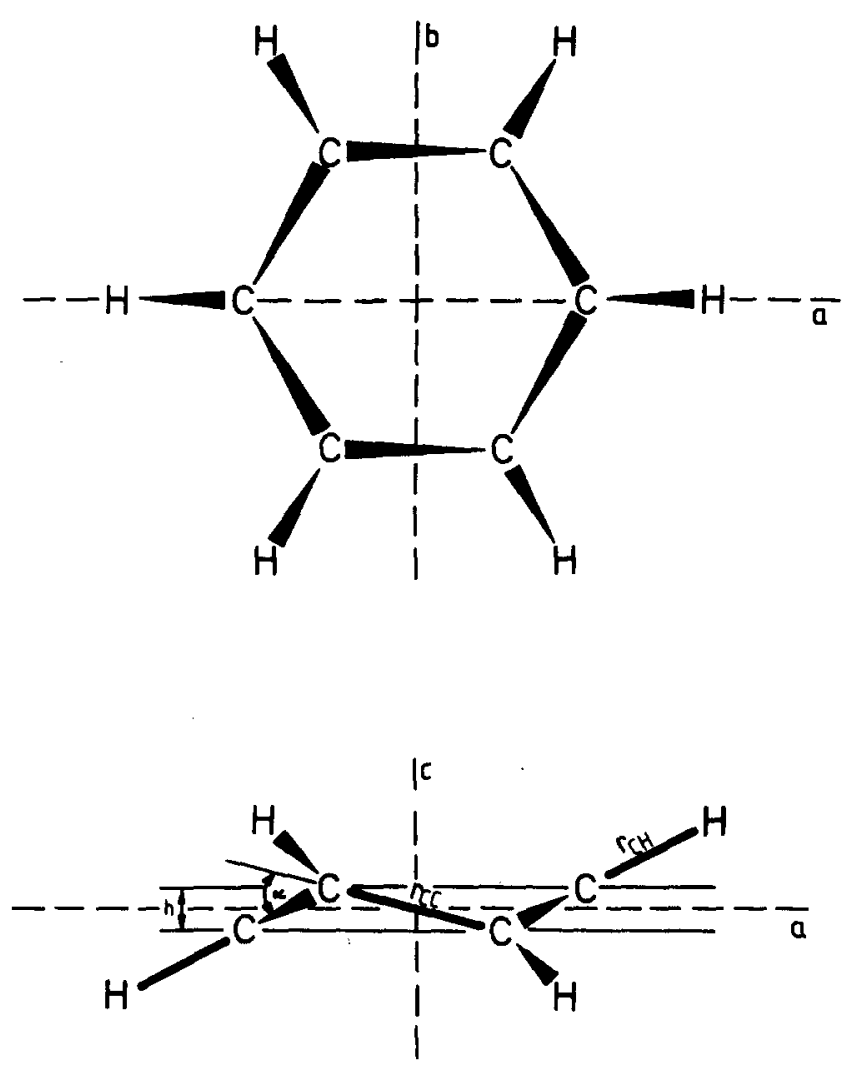

FIG. 6. Effective structure of the $\mathrm{C}_{6} \mathrm{H}_{6}$ molecule as found from our experimental results. $\alpha$ is the angle between the bent planes of the molecule and $h$ is the distance of the two nonidentical parallel planes containing every second $\mathrm{C}$ atom.

This leads to the following new structural characteristics of the benzene molecule in the excited state:

(i) $\mathrm{C}_{6} \mathrm{H}_{6}$ is to a very high degree a symmetric top molecule in the first electronically excited state, i.e., it has an at least threefold rotational axis.

(ii) $\mathrm{C}_{6} \mathrm{H}_{6}$ has an inertial defect of -0.154 (amu $\AA$ ) in the $14^{1}$ state. With the numerical values for $B^{\prime}$ and $C^{\prime}$ we are now able to calculate formally an effective structure of the molecule in the excited state. It has, however, to be examined whether this formally calculated structure resembles the true equilibrium structure of the molecule.

A computer calculation of the rotational constants of the benzene molecule was performed for given positions of the atoms. Starting with an estimated configuration, this calculation was then automatically repeated until the calculated values of $B$ and $C$ fit best to the experimentally observed values. The underlying model is shown in Fig. 6. The chair form of the benzene molecule is characterized by the distance $h$ of the two parallel planes each containing three $\mathrm{C}$ atoms and being a measure of the displacement of the $\mathrm{C}$ atoms from the original molecular plane. An additional result is the angle $\alpha$ between the bent planes of the molecule. Since in this work no is otopic measurements are available, we are not able to determine the $\mathrm{CH}$ bond length $r_{\mathrm{CH}}$ independently. This is presumably of minor importance for possible nonplanarity. For the calculation it is assumed that the $\mathrm{CCCH}$ plane is conserved in the nonplanar bent benzene molecule. As a value for the $\mathrm{CH}$ bond length the result of Ref. 9 is taken, found from analysis of the same band in the Doppler-limited two-photon spectrum. The results of our calculation are summarized in Table II. Even though the $\mathrm{C}$ displacement from the original molecular plane is only $\pm 21 \mathrm{~mA}$, this displacement is comparable with the increase of the $\mathrm{CC}$ bond length $r_{\mathrm{CC}}$ in the first excited state of $35 \mathrm{~mA}$. This out-of-plane displacement, however, gives rise only to small changes of the moments of inertia and hence is more difficult to observe in the UV spectrum than the known increase of the $\mathrm{CC}$ bond length which has a more profound effect on $B$.

Finally, it is of interest to discuss whether the inertial defect is produced by vibration-rotation interaction or by a nonplanarity in the equilibrium configuration of the excited state. Unfortunately, the pure electronic transition $B_{2 u}-A_{1,}$ to the first excited state is twophoton forbidden and we are not able to measure the rotational constants without a vibration excited in the $S_{1}$ state. It is, however, unlikely that the $\nu_{14}\left(b_{2 u}\right)$ vibration being an in-plane vibration can produce a mean square displacement of $\mathrm{C}$ atoms as large as $21 \mathrm{~mA}$ from the molecular plane. Hitherto, vibrational effects on the planarity of the benzene molecule have only been studied in the ground state for several Raman allowed vibrations of $a_{1 g}$ and $e_{2 g}$ symmetry ${ }^{17}$ and also for IR active vibrations. ${ }^{18}$ In most cases the planarity condition $C^{\prime \prime}=\frac{1}{2} B^{\prime \prime}$ is satisfied with high accuracy; typically, the variation of $\left(C_{v}^{\prime \prime}-B_{v}^{\prime \prime}\right)$ from the planar value is $1 \times 10^{-5}$, which is nearly one order of magnitude smaller than found in our spectra. These small variations in the ground state are supposed to be the result of the complicated simulation procedure of the rotational structure in the center and in the wings of the infrared or Raman bands rather than the result of a vibrational distortion of the molecule.

Hence, we conclude that the observed inertial defect is neither produced by the excited quanta of $\nu_{14}$ nor by the zero point vibrations and that the inertial defect is also existent in the equilibrium configuration. Hence, the molecule is assumed to be slightly nonplanar in the excited state. Conceivably, the mixing in of the new molecular orbital tends to enforce a chair form for the excited state.

Further indications for this conclusion would be available when the variation of the rotational constants for different vibrations is known. A calculation of the inertial defect produced by vibration-rotation interaction is in progress.

${ }^{1}$ For a review see I. G. Ross, Adv. Chem. Phys. 20, 341 (1971).

${ }^{2}$ For a review see D. H. Levy, Annu. Rev. Phys. Chem. 31, 197 (1980). 
${ }^{3}$ C. S. Parmenter, Adv. Chem. Phys. 22, 365 (1972).

${ }^{4} \mathrm{~F}$. Biraben, B. Cagnac, and G. Grynberg, Phys. Rev. Lett. 32, 643 (1974); M. D. Levenson and N. Bloembergen, Phys. Rev. Lett. 32, 645 (1974).

${ }^{5}$ W. Hampf, H. J. Neusser, and E. W. Schlag, Chem. Phys. Lett. 46, 406 (1977).

${ }^{6}$ L. Wunsch, F. Metz, H. J. Neusser, and E. W. Schlag, J. Chem. Phys. 66, 386 (1977).

${ }^{7}$ L. Wunsch, H. J. Neusser, and E. W. Schlag, Chem. Phys. Lett. 31, 433 (1975)

${ }^{8} \mathrm{~J}$. H. Callomon, T. M. Dunn, and I. M. Mills, Philos. Trans. R. Soc. London Ser. A 259, 499 (1966).

${ }^{9}$ J. R. Lombardi, R. Wallenstein, T. W. Hänsch, and D. M. Friedrich, J. Chem. Phys. 65, 2357 (1976).

${ }^{10} \mathrm{G}$. Herzberg, Molecular Spectra and Molecular Structure (Van Nostrand, Princeton, 1966), Vol. III.
${ }^{11}$ E. Br. Wilson, J. Chem. Phys. 3, 276 (1935).

${ }^{12}$ B. P. Stoicheff, Can. J. Phys, 32, 339 (1954).

${ }^{13}$ M. R. Aliev, Opt. Spectrosk. 33, 858 (1972).

${ }^{14}$ J. M. Dowling, J. Mol. Spectrosc. 6, 550 (1961).

${ }^{15}$ M. R. Aliev, S. I. Subbotin, and V. I. Tyulin, Opt. Spectrosk. 24, 47 (1968).

${ }^{16} \mathrm{H}$. B. Jensen and S. Brodersen, J. Raman Spectrosc. 8, 103 (1979).

${ }^{17}$ A. B. Hollinger and H. L. Welsh, Can. J. Phys. 56, 974 (1978); 56, 1513 (1978).

${ }^{18} \mathrm{~J}$. Kauppinen, P. Jensen, and S. Brodersen, J. Mol. Spectrosc. 83, $161(1980)$.

${ }^{19} \mathrm{H}$. W. Kroto, Molecular Rotation Spectra (Wiley, London, 1975), p. 34 .

${ }^{20} \mathrm{M}$. Robey and E. W. Schlag, J. Chem. Phys. 67, 2775 (1977). 